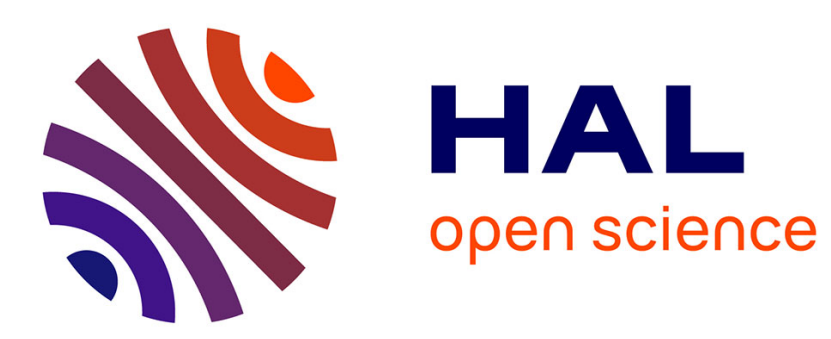

\title{
A NOVEL METHODOLOGY TO MEASURE MASS TRANSFER AROUND A BUBBLE
}

Jessica François, Nicolas Dietrich, Arnaud Cockx

\section{To cite this version:}

Jessica François, Nicolas Dietrich, Arnaud Cockx. A NOVEL METHODOLOGY TO MEASURE MASS TRANSFER AROUND A BUBBLE. Modern Physics Letters B, 2011, 25 (25), pp.1993-2000. 10.1142/S0217984911027236. hal-02352784

\section{HAL Id: hal-02352784 \\ https://hal.science/hal-02352784}

Submitted on 9 Jul 2021

HAL is a multi-disciplinary open access archive for the deposit and dissemination of scientific research documents, whether they are published or not. The documents may come from teaching and research institutions in France or abroad, or from public or private research centers.
L'archive ouverte pluridisciplinaire HAL, est destinée au dépôt et à la diffusion de documents scientifiques de niveau recherche, publiés ou non, émanant des établissements d'enseignement et de recherche français ou étrangers, des laboratoires publics ou privés. 


\title{
A novel methodology to measure mass transfer around a bubble
}

\author{
Jessica FRANCOIS, Nicolas DIETRICH, Arnaud COCKX \\ 1: Université de Toulouse; INSA; INP; UPS; LISBP, 135 Avenue de Rangueil, F-31077 Toulouse, France \\ 2: INRA, UMR792, Ingénierie des Systèmes Biologiques et des Procédés, F-31400 Toulouse, France \\ 3: CNRS, UMR5504, F-31400 Toulouse, France
}

An original approach for accurately measuring the mass transfer of a single bubble rising in a liquid is reported. The approach consists in using PLIF technique (Planar Laser Induced Fluorescence) at micro-scale to capture images of the released oxygen concentration field in the bubble wake. The applicability of the technique for determining mass transfer coefficients in different fluids $(1<R e<100)$ is demonstrated and the relevance of the results is corroborated by established correlations. This direct and non-intrusive technique could be a promising tool to enrich the understanding of mass transfer phenomena.

Gas-liquid mass transfer plays a key role in many industrial applications, such as petrochemical processes or waste water treatment. The performances of these processes are directly linked to the efficiency of the gas transfer phenomenon. Intensive research has been devoted to gasliquid mass transfer for many years but, paradoxically, the classical determination methods present some serious limitations due to the use of a probe for measurement: intrusive and indirect technique, long response time, impact of the liquid media, etc. The aim of the work presented in this letter is to develop a quantitative method for locally and accurately evaluating the mass transferred in the wake of a single bubble rising in a quiescent liquid.

In this purpose Planar Laser Induced Fluorescence (PLIF), which is a non-intrusive alternative method, is used to gain new insight into gas-liquid transfer. PLIF is based on the excitation of a fluorescent dye by light. The fluorescent dye is capable of absorbing light at a specific wavelength $\lambda_{a}$ and then re-emitting part of the received energy in the form of light at a longer wavelength $\lambda_{f}$. The general principle of the PLIF technique consists in illuminating a solution containing a fluorescent dye with a laser sheet and recording the fluorescence of the solution in the illuminated plane with a digital camera. In the presence of certain molecules called quenchers, the fluorescence of the dye is inhibited ${ }^{1,2}$. The fluorescence intensity decreases linearly with the increase of the quencher concentration following the Stern-Volmer equation:

$$
\frac{I_{Q}}{I_{0}}=\frac{1}{1+K_{S V}[Q]}
$$

with $K_{S V}$ the Stern-Volmer constant $\left(\mathrm{L}^{\mathrm{mg}} \mathrm{m}^{-1}\right), I_{Q}$ the fluorescence intensity in the presence of the quencher and $I_{0}$ this intensity in the absence of the quencher. Previous works have shown the possibility of instantaneously visualizing the concentration field in the wake of a single bubble using the PLIF method. Visualization of the oxygen transferred in the wake of a rising bubble ${ }^{3,4,5,6}$ have been obtained, as has the carbon dioxide concentration distribution around a single droplet $^{7,8}$. However, the PLIF technique reveals some limits due to light reflection and refraction on the surface of the bubble. A white ring is systematically observed around the bubble, the bubble casts a shadow, and there is a high intensity contrast between the left and right sides of the image. The optical issues make heavy image processing necessary, leading to less precise information. In addition, the information collected from the image is only valid for a lateral section of the bubble wake and cannot be extended to the whole bubble to estimate the mass transfer. Considering the results obtained in the previous works, the PLIF technique appears quite promising despite obvious optical limitations. We decided to use PLIF as the mass transfer measuring tool but to implement a new optical set-up where the bubble would not interfere with the laser.

(a)
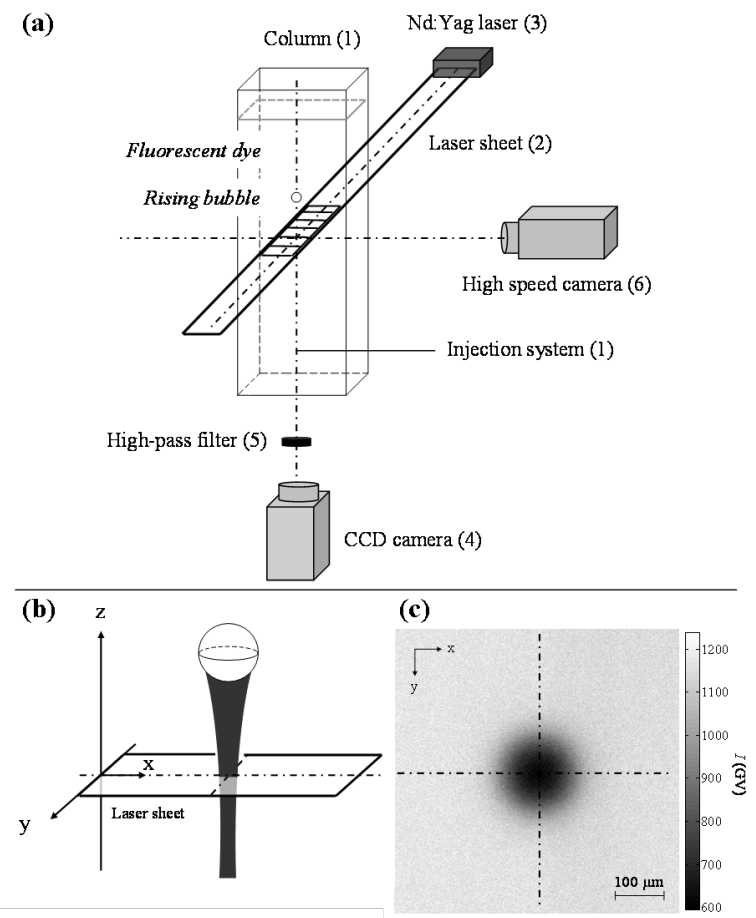

FIG. 1. (a) Experimental set-up. (b) Schematic representation of the bubble wake crossed by the laser sheet in the xy plane. (c) Raw fluorescence image expressed in gray values of a bubble wake cross-section at $z=3 d_{B}$ (System 6). The image corresponds to the intersection of the dashed lines in the $x y$ plane in (b).

The experimental set-up (Fig. 1(a)) consisted of a transparent glass column $\left(100 \times 100 \times 300 \mathrm{~mm}^{3}\right)$ with one side fitted with an injection system (1) to form bubbles. The injection system was composed of a very thin glass capillary with a diameter of the order of $100 \mu \mathrm{m}$ and an automatically controlled syringe (Harvard Apparatus PHD 2000). The column was filled with a solution containing a fluorescent dye. A laser sheet (2) was produced by an Nd: Yag laser (3) (Quantel, USA, $\lambda_{a}=532 \mathrm{~nm}, 10 \mathrm{~Hz}, 2 \times 200 \mathrm{~mJ}$ ) in the xy plane. A CCD camera (4) (Imager Intense, LaVision, Germany, 12 bits, $10 \mathrm{~Hz}, 1040$ x 1376 pixels $^{2}$ ) was placed under the column along the $z$ axis, i.e. perpendicularly to the laser sheet, to record the fluorescence produced by the 
solution. An objective of $105 \mathrm{~mm}$ and three teleconverters were fitted to the camera to reach a spatial resolution of around $2.3 \mu \mathrm{m}$.pixel ${ }^{-1}$. A $570 \mathrm{~nm}$ high-pass filter (5) was also added to film only the fluorescence. The laser sheet and the CCD camera were synchronized by a Programmable Trigger Unit (LaVision, Germany). A high-speed camera (6) (PCO 1200, Germany, 10 bits, $770 \mathrm{~Hz} 1024$ x 1280pixels ${ }^{2}$ ) was placed on the $y$ axis to film the rising bubble at the level of the laser sheet. An objective of $60-\mathrm{mm}$ was fitted to the camera and provided a spatial resolution of around 56 $\mu$ m.pixel ${ }^{-1}$. A ruthenium complex $\mathrm{C}_{72} \mathrm{H}_{48} \mathrm{~N}_{8} \mathrm{O}_{6} \mathrm{Ru}$ (Nanomeps, France) was used as the fluorescent dye at a concentration $[R u]$ of $30 \mathrm{mg} \cdot \mathrm{L}^{-1}$. For each experiment, a single bubble of pure oxygen was formed in an immobile and previously deoxygenated solution. Two bubble sizes were formed: $\mathrm{d}_{\mathrm{B}} \approx 1.5 \mathrm{~mm}$ and $\mathrm{d}_{\mathrm{B}} \approx 0.7 \mathrm{~mm}$. The experimental conditions are detailed in TABLE I. The liquids, of density similar to that of water, were systematically completed by $20 \% \mathrm{w} / \mathrm{w}$ of ethanol to solubilize the ruthenium complex.

TABLE I.Hydrodynamic conditions and mass transfer results for the six set of experiments.

\begin{tabular}{|c|c|c|c|c|c|c|c|c|}
\hline 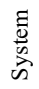 & 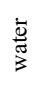 & $\begin{array}{l}\overrightarrow{0} \\
\stackrel{0}{0} \\
\stackrel{0}{b 0}\end{array}$ & $\mu$ & $d_{B}$ & $U_{B}$ & $R e$ & $k_{L}$ & Flux \\
\hline & \multicolumn{2}{|c|}{$\% \mathrm{w} / \mathrm{w}$} & $\begin{array}{l}10^{-3} \\
\text { Pa.s }\end{array}$ & $\mathrm{mm}$ & $\mathrm{m} \cdot \mathrm{s}^{-1}$ & & $10^{-4} \mathrm{~m} \cdot \mathrm{s}^{-1}$ & $10^{-3} \mathrm{~g} \cdot \mathrm{m}^{-2} \cdot \mathrm{s}^{-1}$ \\
\hline 1 & 0 & 80 & 58 & 1.88 & 0.047 & 1.8 & 0.18 & 0.379 \\
\hline 2 & 20 & 60 & 7.0 & 1.29 & 0.117 & 26 & 1.22 & 3.03 \\
\hline 3 & 20 & 60 & 7.0 & 0.72 & 0.032 & 3.4 & 0.46 & 1.13 \\
\hline 4 & 40 & 40 & 2.6 & 1.30 & 0.203 & 107 & 2.92 & 8.48 \\
\hline 5 & 40 & 40 & 2.6 & 0.78 & 0.071 & 22 & 1.47 & 4.26 \\
\hline 6 & 80 & 0 & 1.0 & 0.72 & 0.110 & 74 & 4.31 & 16.5 \\
\hline
\end{tabular}

In the new PLIF configuration, the laser sheet was perpendicular to the bubble trajectory (Fig. 1(b)). Crosssections of the bubble wake were then obtained from the CCD camera images every $0.1 \mathrm{~s}$ (Fig. 1(c)). The information collected on the transfer was thus pertinent at a given section $z$ of the bubble wake and the optical problems were avoided since the bubble did not appear on the images. The exploitation of the high speed camera images allowed the bubble diameter, $d_{B}$, and velocity, $U_{B}$, to be determined together with the distance, $z$, between the laser flashes and the bubble. The fluorescence images recorded with the CCD camera allowed the characterization of the oxygen concentration fields in the bubble wake cross-sections. For an image, the information was initially obtained in gray values and then converted into concentration with the appropriate calibration curve. Figure 2(a) displays four cross-sections with approximately $100 \mathrm{~d}_{\mathrm{B}}$ space between them. It appears that the oxygen concentration fields are perfectly symmetric. They form a concentric, circular gradient of oxygen with the highest concentrations at the centre of the spot. The lateral oxygen concentration profile in the bubble wake is reconstructed from the successive cross-sections in Fig. 2(b) and the elevated concentration profiles associated with the four cross-sections are displayed in Fig. 2(c). On this last figure, the diffusion phenomenon is clearly visible, with a significant flattening and widening of the Gaussian profiles with the distance $z$. From this, a mathematical approach was developed to quantify mass transfer. The approach is based on the implementation of a general oxygen mass balance defined on a closed geometric contour $\partial \mathcal{D}$ can be described by Eq. 2 :

$$
\frac{d m_{O_{2}}}{d t}=-\oiint_{\partial D}\left(\vec{U}-\vec{U}_{\partial D}\right)\left[\left[O_{2}\right] \cdot \vec{n} \cdot d S-\oiint_{\partial D} \overrightarrow{j_{O_{2}}} \cdot \vec{n} \cdot d S\right.
$$

The first term on the right-hand side of Eq. 2 represents the net convective transport, which tends to zero far from the bubble, where the bubble-induced velocity field is drastically dissipated. The second term represents the net oxygen flux transferred by the bubble. It can be assumed that this diffusive transport is negligible in the distant wake in comparison to the interfacial mass transfer.
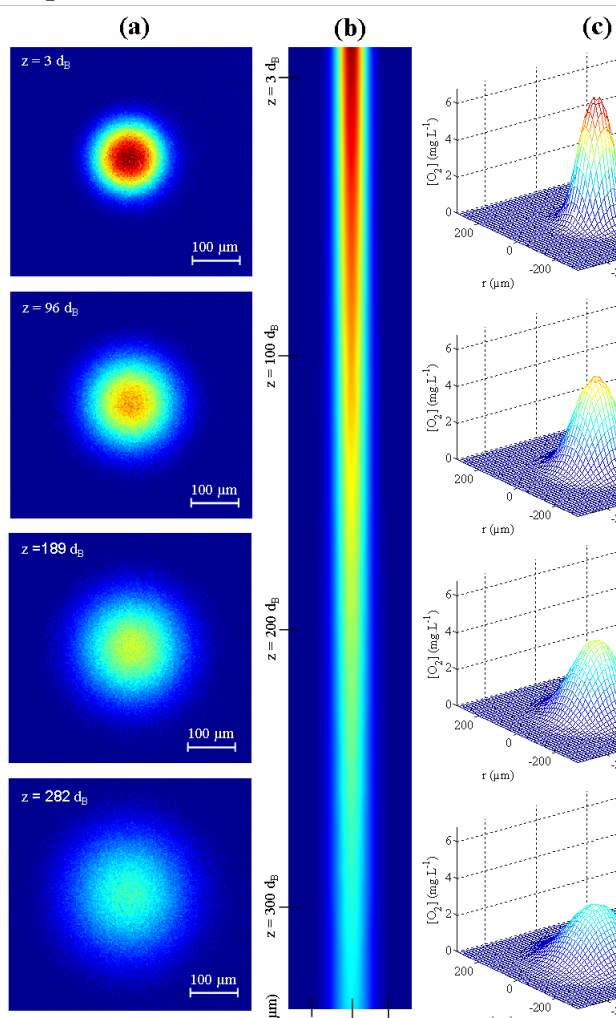

(b)

(c)

$\left[\mathrm{O}_{2}\right]\left(\mathrm{mg} \cdot \mathrm{L}^{-1}\right)$

FIG. 2. (a) Concentration fields of successive bubble wake cross-sections at $z=3 d_{B}, z=96 d_{B}, z=189 d_{B}$ and $z=282 d_{B}$ (System 6). (b) Reconstitution of the lateral oxygen concentration profile in the bubble wake from 50 cross-sections. (c) Elevated oxygen concentration fields in the bubble wake cross-sections at $z=3 d_{B}, z=96 d_{B}, z=189 d_{B}$ and $z=282$ $d_{B}$

In the case of a rising bubble in a quiescent liquid, Eq. 2 can then be reduced to:

$$
\frac{d m_{O_{2}}}{d t}=-\oiint_{\text {Bubble }} \overrightarrow{j_{O_{2}}} \cdot \vec{n} \cdot d S=J_{B}
$$

The oxygen transferred by a single bubble $J_{B}$ can then be tracked by the accumulation in the distant wake. It can also be demonstrated by mass balance that the oxygen accumulation term is defined as: 


$$
\frac{d m_{O_{2}}}{d t}=\iint \vec{U}_{B} \cdot\left[O_{2}\right] \cdot \vec{n} \cdot d S
$$

where $\vec{U}_{B}$ is the bubble velocity and $\left[\mathrm{O}_{2}\right]$ is the oxygen concentration field in a given cross-section. In the case of a measured section normal to the bubble velocity configuration, the oxygen flux will be estimated by:

$$
\text { Flux }=\frac{J_{B}}{\pi \cdot d_{B}{ }^{2}}=\frac{U_{B}}{\pi \cdot d_{B}{ }^{2}} \int_{0}^{\infty} \int_{0}^{\infty}\left[O_{2}\right] d x d y
$$

The oxygen concentration surface $\left[\mathrm{O}_{2}\right](x, y)$ in $\mathrm{Eq} 5$. is extracted from the bubble wake cross section images. An example of elevated oxygen concentration surfaces at different distances $z$ is plotted in Fig. 3(c). These experimental surfaces are very similar and can be superimposed to an axis-symmetric Gaussian curve as:

$$
\left[O_{2}\right] \approx\left[O_{2}\right]_{\max } \cdot e^{-\left(\frac{x+y}{\sigma}\right)^{2}}
$$

To eliminate the experimental noise at large radial distance far from the bubble trajectory, an orthoradial average is done to obtain a radial profile of oxygen $\left[\mathrm{O}_{2}\right](r)$ for each distance $\mathrm{z}$ and the flux is finally estimated by:

$$
\text { Flux }=\frac{2 \cdot U_{B}}{d_{B}{ }^{2}} \int_{0}^{\infty}\left[O_{2}\right] \cdot r \cdot d r
$$

The mass transfer around a single bubble was quantified in six hydrodynamic conditions $(1<R e<100)$. The flux values calculated at successive distances $z$ from the bubble have an asymptotic behavior. The values stabilize only at a sufficiently large distance $z_{c}$ from the bubble. This behavior confirms the hypothesis that, at a certain distance $z$, the wake is no longer disturbed by the bubble passing. The flux can then be estimated as the average flux values from $z=z_{c}$. Mass transfer coefficients $k_{L}$ can be deduced from the flux as described in Eq. 8:

$$
k_{L}=\frac{\text { Flux }}{\left[O_{2}\right]_{\text {sat }}-\left[O_{2}\right]_{\text {ref }}}
$$

Where $\left[\mathrm{O}_{2}\right]_{\text {sat }}$ is the oxygen concentration at saturation and $\left[\mathrm{O}_{2}\right]_{\text {ref }}$ the oxygen concentration in the solution before the bubble passes. The reproducibility of the measurements was checked by repeating the same measurement in the same experimental conditions. The standard deviation of the measured flux was of the order of $3 \%$. The experimental results are presented in TABLE I and shows that the oxygen transfer increase with water/glycerol fraction for similar bubble size (experiments 1-2-4 and 3-5-6) and with bubble size for a same fluid (experiments 2-3 and 4-5).

These mass transfer coefficients were compared to the mass transfer coefficients calculated from the Sadhal and Johnson (1983) exact solution that predict the normalized drag coefficient $C_{D}^{*}$ for a partially contaminated rising bubble. As proposed by Dani (2007) from direct numerical simulation and as in the experimental work of Takemura \& Yabe (1999), this relation fit well the normalized mass transfer coefficient by:

$$
k_{L}^{*}=1-\sqrt{1-C_{D}^{*}}
$$

It was found that the experimental values are in a good agreement with the model as shown in Fig. 3. These results clearly validate the order of magnitude of the experimental quantitative measurements.

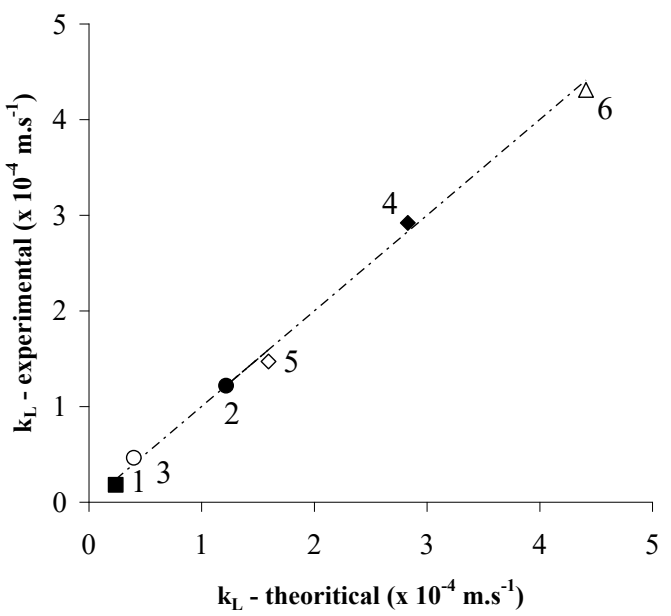

FIG. 3. Parity diagram: experimental $k_{L} v s$. theoretical $k_{L}$ for the six hydrodynamic conditions. $\circ$ : small bubbles $\left(d_{B} \approx 0.7 \mathrm{~mm}\right) \bullet:$ large bubbles $\left(d_{B} \approx 1.5 \mathrm{~mm}\right)$.

To conclude, the approach developed here, and reported for the first time in the literature, uses PLIF in a new optical configuration for the micro-scale quantification of the mass transferred by a single bubble rising. This is the first report to demonstrate the $\mathrm{k}_{\mathrm{L}}$ measurement of oxygen mass transfer by the PLIF method. The determination of the flux only requires the knowledge of the oxygen concentration field present in a cross-section of the bubble wake, and the bubble diameter and velocity. The mass transfer deduced from established theories and that obtained experimentally are in a good agreement, attesting the relevance of the method. The application of the technique to different media could improve and enlarge the current mass transfer models and so become a useful tool for mass transfer analysis.

Compared with the other techniques for the $\mathrm{k}_{\mathrm{L}}$ measurement, there are several prominent advantages in this method. First, particularly, the time-response of this method is excellent. The measurement in the bubble wake is instantaneous and the bubble passage is short $(\sim \mathrm{s})$ rather than longer time for other techniques.

Since $\mathrm{k}_{\mathrm{L}}$ is sensitive to the viscosity as well as the physicochemical properties, this method is not affected by probes limitations as classical methods in theses medium. One of demerits of this method is that it requires the addition of a dye molecule to induce the fluorescence. However, it does not require complicated procedure and it may not be so serious limitation. We believe that this technique will be frequently used for characterizing complex fluids in future.

The support of the French National Research Agency (Project $0_{2}$ STAR) and the FERMaT federation is gratefully acknowledged. 


\section{References:}

${ }^{1}$ J.R. Albani, TEC\&DOC (Paris), pp. 1-137 (2001).

${ }^{2}$ G.D. Geddes, Measurement Science and Technology 12, r53-r88 (2001).

${ }^{3}$ A. Dani, P. Guiraud, and A. Cockx, Chemical Engineering Science 62, 7245-7252 (2007).

${ }^{4}$ O. Bork, M. Schlueter, S. Scheid, and N.Raebiger, FluiPhysics and Heat Transfer for Macro- and Micro-Scale Gas-Liquid and Phase-Change Flows, 11-16 november (2001).

${ }^{5}$ S. Roy and S.R. Duke, Review of Scientific Instruments, v. 71-9 (2000).

${ }^{6}$ S. Roy and S.R. Duke, Exp. Fluids 36, 654-662 (2004).

${ }^{7}$ M. Stöhr, J. Schanze, and A.Khalili, Exp Fluids 47, 135143 (2009).

${ }^{8}$ S. Someya, S. Bando, Y. Song, B. Chen, and M. Nishio, International Journal of Heat and Mass Transfer 48, 25082515 (2005).

${ }^{9}$ Sadhal S.S. and R.E. Johnson, Stokes flow past bubbles and drops partially coated with thin films. Part 1, Stagnant cap of surfactant film - exact solution. Journal of Fluid Mechanics, 126 p. 237, (1983).

10 A. Dani, Ph.D. Thesis, Inst. Nat. Sci. Appl. Toulouse, (2007).

${ }^{11}$ Takemura, F. and Yabe, A., Rising speed and dissolution rate of a carbon dioxide bubble in slightly contaminated water. Journal of Fluid Mechanics, 378 :319-334, (1999). 